\title{
A Influência da Satisfação Laboral no Bem-estar Subjetivo: Uma Perspectiva Geracional
}

\author{
Cibele Ventura Vieira Satuf* \\ Universidade Federal de Minas Gerais, Belo Horizonte, MG, Brasil \\ Samuel José Fonseca Monteiro \\ Henrique Pereira \\ Graça Esgalhado \\ Rosa Marina Afonso \\ Manuel Loureiro \\ Universidade da Beira Interior, Covilhã, Portugal
}

\begin{abstract}
RESUMO - Os estudos relacionados ao bem-estar subjetivo e à satisfação laboral indicam uma relação entre os constructos. Contudo, a magnitude dessa relação pode ser influenciada por diversos fatores, entre eles, as gerações. O objetivo deste trabalho foi identificar as diferenças e semelhanças nas dimensões da satisfação laboral que influenciam o bem-estar subjetivo entre as gerações. Foram analisados os dados de 1042 sujeitos brasileiros e portugueses: 376 da geração Y, 400 da geração X e 266 baby boomers. As análises realizadas mostraram diferenças entre as dimensões da satisfação laboral que atuam como preditores do bem-estar subjetivo e indicaram diferenças na magnitude da relação entre as variáveis. Os resultados sugerem diferenças geracionais na relação entre satisfação laboral e bem-estar subjetivo.
\end{abstract}

Palavras-chave: satisfação laboral, bem-estar subjetivo, gerações.

\section{The influence of job satisfaction on subjective well-being: A generational perspective}

\begin{abstract}
Studies related to subjective well-being and job satisfaction indicate a relationship between the two constructs. However, the magnitude of this relationship can be influenced by several factors, including differences among generations. The aim of this study was to identify the differences and similarities in dimensions of job satisfaction that influence subjective well-being among generations. Data included 1,042 Brazilian and Portuguese subjects: 376 of generation Y, 400 of generation $\mathrm{X}$, and 266 baby boomers. Analysis showed differences between the dimensions of job satisfaction that predicted subjective well-being and indicate differences in the magnitude of the relationship between the variables. Results suggest generational differences in the relationship between job satisfaction and subjective well-being.
\end{abstract}

Keywords: job satisfaction, subjective well-being, generations.

O trabalho é visto como fonte de identificação e autoestima, um meio para se atingir um sentimento de participação nos objetivos da sociedade (Navarro \& Padilha, 2007), por meio da produção de bens e serviços voltados para a satisfação das necessidades sociais (Ribeiro, 2013). Os estudos realizados por Blanch em 1990 indicaram que o trabalho funcionava ainda como um importante fator de estruturação da vida psíquica, sociopolítica e cultural, ao possibilitar salário, status, identidade, direitos e deveres, certeza e segurança (Goulart, 2009). Assim, valorizar a satisfação no trabalho (ST) torna-se importante na vida dos indivíduos e organizações, já que ela possui potencial impacto tanto no bem-estar pessoal quanto na saúde organizacional.

Considerando a importância assumida pelo trabalho na vida dos indivíduos, podemos inferir que a sua avaliação, quer positiva, quer negativa, exercerá, potencialmente, influência na satisfação com a vida como um todo (Russell, 2008). Tal

* Endereço para correspondência: Departamento de Psicologia e Educação, Faculdade de Ciências Sociais e Humanas, Universidade da Beira Interior, Estrada do Sineiro, S/N Covilhã, Portugal. CEP: 6200. E-mail: cibelesatuf@gmail.com relação poderá, contudo, diferenciar-se consoante o papel que o indivíduo atribui ao trabalho, papel este que tende a ser diferente entre as gerações X, Y e Baby Boomers. Dessa maneira, o objetivo central deste artigo é compreender a maneira pela qual a satisfação laboral e o bem-estar subjetivo se influenciam, tendo-se em conta os sujeitos dessas três gerações.

Desde as primeiras décadas do século XX, muito se tem falado a respeito da ST, especialmente entre gestores e estudiosos do comportamento organizacional. O constructo foi tratado inicialmente como algo relacionado com a motivação (Siqueira, 2008) e, posteriormente, passou a remeter para a atitude, compreendendo a avaliação que o sujeito faz sobre diversos elementos de seu trabalho (Alcobia, 2001), avaliação esta capaz de predizer comportamentos organizacionais (Traldi \& Demo, 2012). Sob essa perspetiva, organizações formadas por trabalhadores satisfeitos tendem a possuir maior eficácia nos resultados do que aquelas formadas por trabalhadores insatisfeitos (Hellriegel \& Slocum, 2011). Há, ainda, uma corrente que possui na sua base questões sociais e humanistas e que relaciona a ST à proteção da saúde e do bem-estar dos trabalhadores, de modo que o objetivo das 
organizações passaria a ser a redefinição do trabalho, com vistas a torná-lo rico e desafiador, aumentando os níveis de satisfação (Martins \& Santos, 2006).

Uma das definições de ST mais utilizadas foi proposta por Locke (1976) e trata o constructo como estado emocional positivo, resultante da avaliação das experiências relacionadas ao trabalho. Para Muchinsk (2006), nas últimas décadas, os estudiosos do tema perceberam que o nível de ST de uma pessoa se relacionava tanto ao afeto quanto às condições objetivas propiciadas pelo trabalho, geralmente representadas pelas facetas ou dimensões da ST. De acordo com Sánchez-Sellero, Sánchez-Sellero, Cruz-Gonzáles e Sánchez-Sellero (2014), podemos considerar a ST como um constructo multidimensional, que envolve a avaliação das características do indivíduo, do ambiente de trabalho ou a combinação de ambas, e da qual resulta uma percepção de que o trabalho cumpre as expectativas da pessoa.

Estudos sugerem que a ST pode influenciar o comportamento dos indivíduos nas organizações, alterando índices de produtividade (Sánchez-Sellero et al., 2014), comprometimento organizacional (Folami, Asare, Kwesiga, \& Bline, 2014) e turnover (Zopiatis, Constanti, $\&$ Theocharous, 2014). Além disso, a ST pode influenciar a esfera extralaboral, podendo favorecer ou comprometer índices de bem-estar (Bruk-Lee, Khoury, Nixon, Goh, \& Spector, 2009; Satuf et al., 2016) e felicidade (Bowling, Eschleman, \& Wang, 2010)

Neste estudo, interessa-nos explorar e compreender a relação entre ST e bem-estar subjetivo (BES), que pode ser definido como um termo amplo utilizado para descrever o nível de bem-estar vivenciado pela pessoa, conforme a avaliação subjetiva que ela faz de sua vida. Tal avaliação pode ser positiva ou negativa e inclui julgamentos e sentimentos sobre a satisfação com a vida, interesse e comprometimento, reações afetivas, além de avaliações de seu trabalho, relacionamentos, saúde, propósito de vida, entre outros domínios (Diener \& Ryan, 2009). Abrange desde situações específicas e concretas a globais e abstratas, experiências momentâneas versus julgamento global de vida (Kim-Prieto, Diener, Tamir, Scollon, \& Diener, 2005).

Além da vertente emocional (afetos), o BES inclui uma componente cognitiva, conhecida como satisfação com a vida, que envolve julgamentos globais de satisfação com a vida e com domínios específicos (Gouveia, Fonsêca, Lins, Lima, \& Gouveia, 2008; Galinha \& Pais-Ribeiro, 2005a). A dimensão cognitiva, os afetos positivos (AP) e os afetos negativos (AN) formam constructos distintos (Diener, Suh, Lucas, \& Smith, 1999), mas que se inter-relacionam (Galinha \& Pais-Ribeiro, 2005b).

Entre a população adulta, é expectável que a ST e o BES estejam conectados, já que um adulto tende a passar no trabalho mais de metade das horas em que está acordado (Judge \& Klinger, 2008). Entretanto, a maneira como essas variáveis se relacionam ainda é alvo de estudos e controvérsias. Os principais modelos teóricos que explicam o sentido da influência entre o bem-estar e as diferentes esferas e acontecimentos da vida são chamados de bottom up, ou base-topo, e top down, ou topo-base.

A abordagem bottom up sugere que a satisfação imediata de necessidades tende a produzir o bem-estar, de modo que os acontecimentos positivos vivenciados pela pessoa sejam internalizados e produzam a experiência de bem-estar. Segundo esse modelo, quanto mais eventos positivos a pessoa vivenciar, maiores níveis de BES reportará (Diener \& Ryan, 2009). A percepção do bem-estar como a soma de vários pequenos prazeres oriundos dos diversos domínios está assente na teoria do bottom-up. Durante o processo de avaliação da vida, os domínios são ponderados e os resultados desse julgamento são agregados em uma avaliação integral de bem-estar (Schimmack, Diener, \& Oishi, 2009), em um processo intermediado pelos afetos (Lucas \& Diener, 2009). Desse modo, quando o indivíduo percebe que a frequência de momentos felizes é maior, a tendência é que ele denote satisfação e bem-estar (Diener, 2009).

A abordagem top-down preconiza que a pessoa tenha propensão a julgar os acontecimentos como positivos ou negativos conforme suas experiências e bagagens pessoais, de modo que a atitude, e não os eventos, seria a causadora do bem-estar (Diener \& Ryan, 2009). Assim, o indivíduo demonstra uma propensão a avaliar os momentos de maneira positiva porque é feliz, e, segundo Diener (2009), a personalidade tende a influenciar o tom afetivo que a pessoa utiliza ao avaliar as experiências.

Neste trabalho, consideramos que a abordagem bottomup seja a mais adequada ao estudo das variáveis em questão e elencamos a avaliação da esfera laboral como um potencial fator de influência nessa relação, já que o trabalho confere identidade e reconhecimento às pessoas, permite o preenchimento de suas necessidades individuais, possibilita a organização de rotinas e o engajamento com causas coletivas (Andersen, 2009), sendo um elemento gerador de sentido de vida (Bitencourt, Onuma, Piccinini, Moreira, \& Severo, 2014).

Entre as possíveis formas de relação entre ST e BES, alguns dos investigadores da área em questão especularam três modelos: spillover, segmentação e compensação. A relação através do formato spillover pressupõe que o estado de satisfação com as experiências no trabalho será generalizado para as experiências de vida e vice versa, ou seja, a relação é bidirecional (Lent et al., 2005). Nesse caso, implica-se que as emoções, atitudes e comportamentos de um dos domínios produzam efeito no outro (Georgellis, Lange, \& Tabvuma, 2012), em um sentido de reciprocidade, de modo que as variáveis ST e BES tenham uma correlação positiva (Nielsen, Smyth, \& Liu, 2011).

O modelo de segmentação pressupõe que as experiências de trabalho e de vida sejam tratadas em "compartimentos" diferentes e, portanto, não exerçam influência entre si (Judge \& Klinger, 2008; Georgellis et al., 2012). Nesse modelo, ST e BES revelam distribuições substancialmente diferentes (Georgellis \& Lange, 2011) e denotam correlação muito fraca ou não significativa (Judge \& Watanabe, 1994).

O modelo da compensação pressupõe que o indivíduo procure compensar sua insatisfação em um domínio por meio da busca de satisfação com outras esferas da vida e vice-versa (Judge \& Klinger, 2008; Kehinde, 2011). Sob essa óptica, os dois constructos teriam uma relação inversa ou negativa (Newman, Nielsen, Smyth, \& Hooke, 2015; Nielsen et al., 2011). 
Neste artigo, partimos de uma questão inicial que se centra em analisar as diferenças na relação entre ST e BES entre as gerações formadas pelos Baby Boomers, geração $\mathrm{X}$ e geração $\mathrm{Y}$, considerando a perspectiva bottom-up. Nossas hipóteses, de caráter exploratório, são: H1 - Existem diferenças entre as dimensões da ST que predizem o BES quando a amostra estudada é considerada em função das gerações; e H2 - Há diferenças entre a força do efeito $\left(\mathrm{R}^{2}\right)$ da ST no BES quando se comparam as três gerações. Uma geração pode ser definida como um grupo de pessoas que em um determinado período de tempo compartilha hábitos e cultura, de modo que estabeleçam uma memória coletiva que as integra por um período finito de tempo (Benson \& Brown, 2011). As diferenças entre gerações podem causar impactos na maneira como a personalidade, valores, crenças e expectativas se desenvolvem e tendem a ocorrer devido a influências no ambiente em que se desenvolve a socialização (Macky, Gardner, \& Forsyth, 2008). Ao aplicarmos esse pressuposto no cenário profissional, esperase que as características geracionais influenciem as atitudes, expectativas e avaliações do trabalho (To \& Tam, 2013).

A literatura aponta algumas divergências na definição dos limites de idades que formam as gerações, mas grande parte dos estudos indica que os boomers nasceram entre 1946 e 1964 (Young, Sturts, Ross, \& Kim, 2013); a geração X, entre 1965 e 1980 e a geração Y, de 1981 até o início dos anos 2000 (Gursoy, Maier, \& Chi, 2008; Murray, 2011). Ressalta-se que geralmente a definição do coorte geracional é marcada pelos acontecimentos históricos de relevância de dado país. No Brasil, por exemplo, alguns desses marcos são o pós-guerra, a Era Vargas, e os Anos Dourados, o que resultaria em diferentes períodos para essas gerações (Parry \& Urwin, 2011). Ainda assim, optamos por manter essa divisão geracional por integrar sujeitos de dois países distintos e por considerá-la amplamente difundida em diversos estudos. Além disso, conforme Edmunds e Turner (2005) afirmam, é possível argumentar que os eventos ocorridos no final do século XIX e início do século XX, como a Rosa de Luxemburgo, a Guerra Civil Espanhola e a Segunda Guerra Mundial tiveram efeitos nas gerações ao redor do mundo. As gerações se comunicam, seja através de rádio, TV ou outros meios e interagem através das fronteiras, favorecendo as chamadas gerações globais, que permitem a adoção de um referencial geracional supranacional.

Não visando à generalização ou uniformização, a literatura tem tipificado um conjunto de características ou tendências associadas com especificidades de cada geração. A geração chamada baby boomer tende a valorizar a segurança no trabalho e a denotar maior lealdade às empresas, tende a ser mais tradicional e habituada a autoridades, bem como a demonstrar interesse pelo poder e status ao longo da carreira, indicando, ainda, expectativas por recompensas extrínsecas em troca de seu comprometimento (Cavazotte, Lemos, \& Viana, 2012). A geração $X$ tende a ser caracterizada por indivíduos mais cínicos em relação a figuras de autoridade, individualistas e que buscam equilíbrio entre as esferas pessoal e profissional (Cheeseman \& Downey, 2011). Tendencialmente não acreditam que possuam dívidas para com a organização e, portanto, costumam ser menos leais às empresas e mais propensos a mudar de organização em busca da satisfação de suas ambições profissionais (Benson \& Brown, 2011). Por fim, os representantes da geração $\mathrm{Y}$, ou Millennials, costumam ser mais afeitos à comunicação textual, mais participativos no ambiente de trabalho (Cheeseman \& Downey, 2011), além de mais desejosos de feedback e recompensas imediatas pelo trabalho desenvolvido (Young et al., 2013).

Diversos estudos foram conduzidos no sentido de verificar a diferença entre os níveis de satisfação laboral reportados pelas gerações. Alguns apontaram para a maior satisfação laboral entre os Millenials em comparação às gerações mais velhas (Kowske, Rasch, \& Wiley, 2010; Twenge, 2010), mas outros evidenciaram maior satisfação laboral entre os boomers (Benson, \& Brown, 2011; Costanza, Badger, Fraser, Severt \& Gade, 2012). Ainda no que tange à comparação entre gerações, estudos apontam para maiores níveis de BES entre os boomers, quando comparados com as gerações mais novas (Blanchflower \& Oswald, 2008; García, 2015). O modo como a ST e o BES se relacionam depende, contudo, de alguns fatores, como, por exemplo da importância atribuída ao trabalho (Henne \& Locke, 1985), de modo que os efeitos tendem a ser mais intensos entre aqueles que atribuem ao trabalho um papel mais forte de centralidade, como ocorre com a geração dos baby boomers.

\section{Método}

\section{Participantes}

No total, participaram do estudo 1042 sujeitos, de origem brasileira e portuguesa. Entre eles, 376 representantes da geração Y (17 a 33 anos); 400 da geração X (35 a 49 anos) e 266 baby boomers ( 50 a 68 anos).

\section{Procedimentos}

Os questionários foram enviados aos respondentes prioritariamente através de meio eletrônico, no período de outubro de 2014 e fevereiro de 2015 . O formulário utilizado continha informações iniciais sobre a contextualização dos objetivos da investigação, bem como os termos de consentimento livre e esclarecido. Aos indivíduos convidados a participar, foram garantidos o total anonimato e a confidencialidade dos dados recolhidos.

\section{Instrumentos}

Por meio do questionário sociodemográfico foram coletadas informações sobre idade, gênero, escolaridade, estado civil e situação profissional.

A ST foi medida por meio da Escala de Satisfação no Trabalho - EST, versão reduzida, desenvolvida por Siqueira em 1995. O instrumento possui 15 itens, divididos em cinco dimensões: satisfação com os colegas, com o salário, com a chefia, com a natureza do trabalho e com as promoções (Siqueira, 2008). As respostas são obtidas através de uma 
escala do tipo Likert, que varia de 1 (totalmente insatisfeito) a 7 (totalmente satisfeito). Neste estudo, os valores de consistência do Alfa de Cronbach variaram entre 0,832 e 0,932 .

O BES foi medido por meio da versão reduzida da escala portuguesa de AP e AN: PANAS-VRP, validada para a população portuguesa por Galinha, Pereira e Esteves (2014). A escala possui 10 itens, sendo 5 para AP e 5 para AN. As respostas são indicadas por meio de uma escala Likert, que varia entre 1 (nada ou muito ligeiramente) e 5 (extremamente). Nesta investigação, os valores do Alfa de Cronbach foram 0,869 e 0,851 .

\section{Análises estatísticas}

A análise de dados foi efetuada com o Statistical Package for Social Sciences (SPSS) versão 22.0. Tendo em consideração as características e a dimensão da amostra, a normalidade da mesma foi testada e verificada. A relativização da normalidade foi justificada por meio do teorema do limite central, que postula que, na medida em que a dimensão da amostra aumenta, a distribuição de sua média tende para a distribuição normal (Dancey \& Reidy, 2011; Maroco, 2007; Tabachnick \& Fidell, 2007). Adicionalmente, foram verificados os valores de assimetria e de curtose, que se situaram entre -1,1 e - 0,02 (assimetria) e -1,2 e - 0,06 (curtose), valores que indicam normalidade aproximada (Field, 2009; Gravetter \& Wallnau, 2014). Nota-se que o maior índice foi verificado na curtose, mas ressalta-se que essa medida não parece afetar de forma significativa a maioria das análises estatísticas (Leech, Barrett, \& Morgan, 2015). Para verificação das dimensões da ST que atuam como preditoras do bem-estar foi utilizada a regressão linear múltipla, método enter, com o intuito de analisar de maneira exploratória os preditores do BES. Por ser um estudo de caráter exploratório, não foi determinado um modelo teórico. Nas análises de regressão, os pressupostos da normalidade, multicolinearidade e homogeneidade nas variâncias foram observados. Previamente foi verificado se havia diferenças entre as médias reportadas pelos respondentes nas variáveis principais, por meio da análise de variância múltipla (MANOVA).

\section{Resultados}

Na Tabela 1, são apresentadas as características sociodemográficas considerando apenas as respostas válidas. Entre os respondentes, 480 (46,1\%) eram homens; $561(53,8 \%)$ eram mulheres e $1(0,1 \%)$ indicou outro. A maioria, 436 (41,9\%), era casada; 323 (31,1\%) eram solteiros; 101 (9,7\%), unidos de fato; 86 (8,3\%), divorciados; $84(8,1 \%)$ indicaram possuir compromisso afetivo com alguém significativo; e $10(1 \%)$ eram viúvos. Em relação à escolaridade, 409 (39,5\%) indicaram possuir pós-graduação/ mestrado; 331 (31,9\%) possuíam licenciatura/bacharelado; $164(15,8)$ possuíam doutoramento/pós-doutoramento; 111 $(10,7)$ indicaram possuir até 12 anos de escolaridade; 17 $(1,6 \%)$, até 9 anos de escolaridade; $3(0,3 \%)$, até 6 anos de

Tabela 1. Caracterização sociodemográfica da amostra $(N=1042)$

\begin{tabular}{|c|c|c|c|}
\hline Variáveis & & $N$ & $\%$ \\
\hline \multirow[t]{3}{*}{ Gênero } & Mulher & 561 & 53,8 \\
\hline & Homem & 480 & 46,1 \\
\hline & Outro & 1 & 0,1 \\
\hline \multirow[t]{6}{*}{ Estado civil } & Casado (a) & 436 & 41,9 \\
\hline & Solteiro (a) & 323 & 31,1 \\
\hline & Unido (a) de fato & 101 & 9,7 \\
\hline & Divorciado (a)/Separado (a) & 86 & 8,3 \\
\hline & Compromisso afetivo com alguém significativo & 84 & 8,1 \\
\hline & Viúvo (a) & 10 & 1 \\
\hline \multirow[t]{7}{*}{ Escolaridade } & Pós-graduação/"Mestrado & 409 & 39,5 \\
\hline & Licenciatura/bacharelato & 331 & 31,9 \\
\hline & Doutoramento/Pós-Doutoramento & 164 & 15,8 \\
\hline & Até 12 anos & 111 & 10,7 \\
\hline & Até 9 anos & 17 & 1,6 \\
\hline & Até 6 anos & 3 & 0,3 \\
\hline & Até 4 anos & 1 & 0,1 \\
\hline \multirow{7}{*}{$\begin{array}{l}\text { Situação } \\
\text { profissional }\end{array}$} & Trabalhador (a) por conta de outrem & 601 & 58 \\
\hline & Trabalhador (a) por conta própria & 162 & 15,6 \\
\hline & Trabalhador (a)-estudante & 159 & 15,3 \\
\hline & Estudante & 52 & 5 \\
\hline & Outra & 35 & 3,4 \\
\hline & Desempregado (a) & 14 & 1,4 \\
\hline & Reformado (a)/Aposentado (a) & 13 & 1 \\
\hline \multirow[t]{3}{*}{ Nacionalidade } & Brasileira & 629 & 61,1 \\
\hline & Portuguesa & 388 & 37,7 \\
\hline & Luso-Brasileira & 13 & 1,2 \\
\hline
\end{tabular}


escolaridade; e $1(0,1 \%)$, até 4 anos de escolaridade. A maior parte era brasileira $(61,1 \%) ; 388$ eram portugueses $(37,7 \%)$; e $13(1,2 \%)$ tinham dupla nacionalidade (luso-brasileira). As idades variaram entre 17 e 68 anos, sendo 36,59 anos a idade média $(D P=12.11), 38$ a mediana e 28 anos a moda.

Em relação à situação profissional, 601 (58\%) eram trabalhadores por conta de outrem; $162(15,6 \%)$, trabalhadores por conta própria; $159(15,3 \%)$ trabalhadores estudantes; 52 (5\%), estudantes; 14 (1,4\%), desempregados; 13 (1,3\%), aposentados; e, por fim, $35(3,4 \%)$ responderam outra.

No âmbito da hipótese 1, que visou analisar a existência de diferenças entre as dimensões da ST que predizem o BES quando a amostra estudada é considerada em função das gerações, a análise MANOVA revelou diferença entre os grupos, Pillai's Trace $=0,93, F=4,79, p<0,001$. A comparação entre as médias de ST e BES indicaram diferenças estatisticamente significativas em todas as dimensões da ST (cf. Tabela 2). Os valores para cada uma das dimensões foram: satisfação com os colegas $F(3,1051)$ $=4,30, p=0,005$; satisfação com o salário $F(3,1051)=8,16$, $p<0,001$; satisfação com a chefia $F(3,1051)=3,65, p=$ 0,012 ; satisfação com a natureza do trabalho $F(3,1051)=$ $14,47, p<0,001$; satisfação com as promoções $F(3,1051)=$ $10,44, p<0,001$. O teste Post-Hoc de $L S D$ indicou diferenças ao compararmos os baby boomers com a geração X e com a geração Y em relação à satisfação com os colegas, com o salário e com as promoções, sendo que os baby boomers foram os mais satisfeitos e a geração $\mathrm{X}$, a menos satisfeita. $\mathrm{Na}$ dimensão natureza do trabalho, também foram encontradas diferenças significativas ao compararmos os baby boomers com a geração X e com a geração Y. Novamente os boomers reportaram maior satisfação, mas os menores níveis de

Tabela 2. Satisfação laboral e bem-estar subjetivo entre gerações utilizando MANOVA e teste post-hoc de LSD

\begin{tabular}{|c|c|c|c|c|}
\hline & $M$ & $D P$ & $\boldsymbol{F}$ & $\begin{array}{c}\text { LSD Pares } \\
\text { (Diferença média, p) }\end{array}$ \\
\hline Colegas & & & $4.30 ; p=0,005^{* *}$ & \\
\hline Baby Boomer & 5,09 & 1,06 & & $1-2\left(0,34 ; \mathrm{p}=<0,001^{* * *}\right)$ \\
\hline Geração X & 4,75 & 1,23 & & $2-3(-0,10 ; p=0,251)$ \\
\hline Geração Y & 4,85 & 1,27 & & $1-3(0,24 ; p=0,013 *)$ \\
\hline Salário & & & 8,$16 ; \mathrm{p}<0,001 * * *$ & \\
\hline Baby Boomer & 4,17 & 1,61 & & $1-2(0,59 ; \mathrm{p}<0,001 * * *)$ \\
\hline Geração X & 3,58 & 1,53 & & $2-3(-0,15 ; p=0,167)$ \\
\hline Geração Y & 3,73 & 1,51 & & $1-3(0,44 ; p=0,002 * *)$ \\
\hline Chefia & & & 3,$65 ; p=0,012^{*}$ & \\
\hline Baby Boomer & 4,95 & 1,39 & & $1-2\left(0,32 ; p=0,006^{* *}\right)$ \\
\hline Geração X & 4,63 & 1,54 & & $2-3\left(-0,24 ; p=0,024^{*}\right)$ \\
\hline Geração Y & 4,87 & 1,44 & & $1-3(0,08 ; p=0,480)$ \\
\hline Natureza do trabalho & & & 14,$47 ; \mathrm{p}<0,001 * * *$ & \\
\hline Baby Boomer & 5,34 & 1,06 & & $1-2(0,52 ; \mathrm{p}<0,001 * * *)$ \\
\hline Geração X & 4,81 & 1,31 & & $2-3(0,04 ; p=0,621)$ \\
\hline Geração Y & 4,77 & 1,24 & & $1-3(0,57 ; \mathrm{p}<0,001 * * *)$ \\
\hline Promoções & & & 10,$44 ; \mathrm{p}<0,001 * * *$ & \\
\hline Baby Boomer & 4,31 & 1,53 & & $1-2(0,60 ; \mathrm{p}<0,001 * * *)$ \\
\hline Geração X & 3,71 & 1,47 & & $2-3(-0,06 ; p=0,585)$ \\
\hline Geração Y & 3,76 & 1,41 & & $1-3(0,54 ; \mathrm{p}<0,001 * * *)$ \\
\hline Afeto positivo & & & 5,$65 ; \mathrm{p}=0,001 * * *$ & \\
\hline Baby Boomer & 20,10 & 3,25 & & $1-2\left(-0,76 ; p=0,006^{* *}\right)$ \\
\hline Geração X & 19,34 & 3,66 & & $2-3(0,34 ; p=0,177)$ \\
\hline Geração Y & 19,00 & 3,44 & & $1-3(1,10 ; \mathrm{p}<0,001 * * *)$ \\
\hline Afeto negativo & & & 11,$53 ; \mathrm{p}<0,001 * * *$ & \\
\hline Baby Boomer & 8,78 & 3,24 & & $1-2(-1,16 ; \mathrm{p}<0,001 * * *)$ \\
\hline Geração X & 9,93 & 3,86 & & $2-3\left(-0,67 ; p=0,016^{*}\right)$ \\
\hline Geração Y & 10,60 & 4,31 & & $1-3\left(-1,83 ; \mathrm{p}<0,001^{* * *}\right)$ \\
\hline
\end{tabular}

Nota: *valores significativos $p<0,05 ; * *$ valores muito significativos $p<0,01 ; * * *$ valores altamente significativos $p<0,001$. Baby Boomer (1): $n=266$; Geração X (2): $n=400$; Geração Y (3): n = 376 
satisfação foram encontrados na geração Y. Na dimensão satisfação com a chefia, foram encontradas diferenças significativas somente entre os boomers e a geração X, sendo esta a que reportou menores níveis de satisfação. Entre as gerações $\mathrm{X}$ e Y, somente foram encontradas diferenças estatisticamente significativas quando se considerou a satisfação com a chefia, na qual a geração Y apresentou a maior média.

A análise referente à comparação dos níveis de BES entre as três gerações apontou diferenças estatísticas significativas nos dois afetos. Os valores foram $F(3,1051)=5,65, p=$ 0,001 para o AP; e $F(3,1051)=11,53, p<0,001$ para o AN. Os boomers revelaram maior presença de AP e menor presença de $\mathrm{AN}$, enquanto a geração Y reportou maior nível de AN e menor índice de AP (cf Tabela 2). O teste Post-Hoc de LSD indicou diferenças estatisticamente significativas na comparação dos boomers com a geração X e entre boomers e geração Y, quando considerado o AP. No caso do AN. foram encontradas diferenças em todas as combinações de grupos. Em ambos os afetos, a geração Y reportou índices menos favoráveis, ao passo que os baby boomers apresentaram índices mais favoráveis.

No âmbito da segunda hipótese, que visou explorar as diferenças entre a força do efeito $\left(\mathrm{R}^{2}\right)$ da ST no BES quando se comparam as três gerações, foram realizadas análises de regressão linear múltipla. Na Tabela 3, estão apresentados os coeficientes de regressão não padronizados (B), os valores de erro padrão de $\mathrm{B}(\mathrm{EPB})$, os coeficientes de regressão padronizados ( $\beta$ ), o $\mathrm{R}^{2}$ e o $\mathrm{R}^{2}$ ajustado.

Na geração $Y$, as dimensões da satisfação com a natureza do trabalho $(\beta=0,392, p<0,001$ e satisfação com os colegas ( $\beta=0,128, p=0,033)$ contribuiram significativamente com $17,9 \%$ da explicação do AP. A satisfação com a natureza do trabalho explicou $4,9 \%$ do $\mathrm{AN}$, nesse caso com coeficiente menor $(\beta=-0,241, p<0,001)$. O valor de $\mathrm{R}$ para a regressão foi significativamente diferente de zero para o AP, $F(5,370)$ $=17,38, p<0,001]$, e para o AN, $F(5,370)=4,88, p<$ $0,001]$. As demais dimensões da ST não contribuíram para a explicação do BES, nessa geração.

Na geração $X$, as dimensões da satisfação com a natureza do trabalho $(\beta=0,482, p<0,001)$ e satisfação com a chefia $(\beta=-0,154, p=0,015)$ contribuíram significativamente com $23,1 \%$ da explicação do AP. A dimensão satisfação com a natureza do trabalho $(\beta=-0,161, p=0,017)$ explicou $5,8 \%$ do AN. O R para a regressão foi significativamente diferente de zero para o AP, $F(5,395)=25,01, p<0,001$, e para o $\mathrm{AN}$, $F(5,394)=5,91, p<0,001]$. As demais dimensões da ST não contribuíram para a explicação do BES, nessa geração.

Já entre os baby boomers, as dimensões da satisfação com a natureza do trabalho $(\beta=0,462, p<0,001)$, satisfação com os colegas $(\beta=0,215, p<0,001)$ e satisfação com o salário $(\beta=-0,132, p=0,049)$ contribuíram significativamente com $28,2 \%$ da explicação do AP, enquanto as dimensões da satisfação com a natureza do trabalho $(\beta=-0,329, p<$ $0,001)$ e satisfação com os colegas $(\beta=-0,162, p=0,018)$ contribuíram significativamente com $16,7 \%$ da explicação do AN. Os valores de R para a regressão foram $F(5,260)=$ $21,83, p<0,001]$ para o AP e $F(5,260)=11,61, p<0,001$ no AN.

\section{Discussão}

Por meio da comparação dos níveis de ST entre as gerações, foi possível identificar que os baby boomers reportaram maiores níveis de satisfação com todas as dimensões, enquanto a geração X reportou os menores níveis de satisfação, exceto na dimensão natureza do trabalho, em que os Millennials reportaram maior insatisfação. Em uma meta-análise acerca de diferenças nas atitudes relacionadas ao trabalho entre gerações, Costanza et al. (2012) identificaram diversos estudos que apontaram as gerações mais velhas como as mais satisfeitas em relação ao trabalho, resultado também confirmado pelas investigações conduzidas por Benson e Brown (2011) e por Young et al. (2013). Alguns estudos mostram que devido à experiência profissional e maior conhecimento do trabalho, os boomers tendem a possuir expectativas mais realistas, o que se reflete em maiores índices de satisfação (Young et al., 2013). Já os mais novos tenderiam a iniciar a carreira com maiores níveis de entusiasmo e expectativas em relação ao trabalho, entretanto, com o passar do tempo, poderiam se deparar com aumento

Tabela 3. Regressão múltipla (método enter) sobre o efeito preditor da satisfação com o trabalho no bem-estar entre gerações

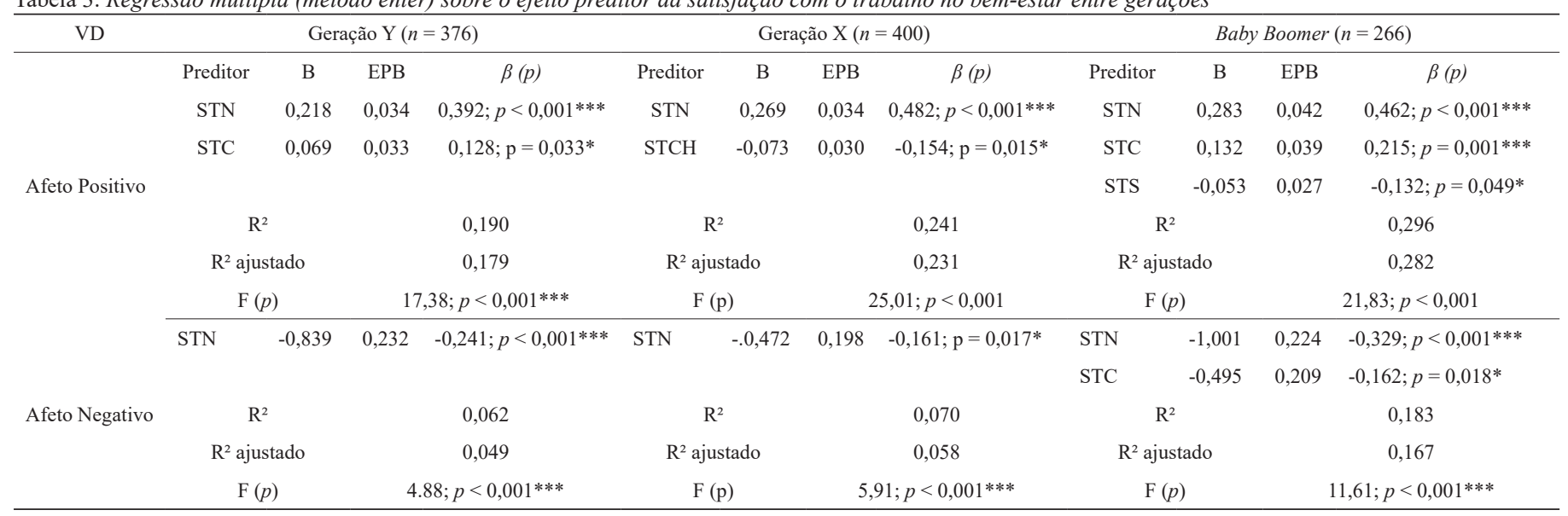

Nota: *valores significativos $p<0,05 ; * *$ valores muito significativos $p<0,01 ; * * *$ valores altamente significativos $p<0,001$. STN $=$ Satisfação com a natureza do trabalho; STC $=$ satisfação com os colegas; STS = satisfação com o salário 
da rotina e com poucas oportunidades de crescimento e mudança, o que causaria um desencorajamento gradual (Warr, 2011).

Em relação ao BES, os resultados mostraram que os mais velhos reportaram maior presença de AP e menor presença de AN, enquanto a geração mais jovem demonstrou menor AP e maior AN. Magai (2008) fez alusão a estudos semelhantes nos quais foi verificado um aumento do AP e declínio do AN com o passar dos anos. Warr (2011) relatou estudos em que os mais velhos manifestaram maior felicidade, apesar de a diferença entre estes e os mais novos ser pequena.

A comparação entre as dimensões da ST que atuaram como preditores do BES mostrou que foi a dimensão da satisfação com a natureza do trabalho a que prestou maior contributo para a explicação do bem-estar. Na geração Y, somente a satisfação com a natureza do trabalho e a satisfação com os colegas influenciaram o AP. Na geração $\mathrm{X}$, o AP foi influenciado pela satisfação com a natureza do trabalho e com a chefia. Já entre os baby boomers, a satisfação com as dimensões natureza do trabalho, colegas e salário influenciaram o AP. Ao analisarmos a influência da satisfação laboral no AN, a dimensão da natureza do trabalho também esteve presente em todos os grupos. Porém, para os boomers, a insatisfação com os colegas também revelou potencial para elevar a presença de experiências emocionais negativas. Adicionalmente, o efeito da ST no BES diferiu entre as gerações, sendo mais forte entre os baby boomers. Assim, a partir dos resultados das análises efetuadas, encontraram-se evidências que apontaram para a rejeição das hipóteses nulas e consequente aceitação das hipóteses $\mathrm{H} 1$ e H2 formuladas.

Os valores e objetivos relacionados ao trabalho tendem a diferir entre as gerações. Os boomers, por exemplo, valorizam menos o status e a geração $\mathrm{Y}$ tende a apreciar mais a liberdade no trabalho (Cennamo \& Gardner, 2008), ao passo que a geração $X$ tende a atribuir um papel de menor centralidade ao trabalho (Brown, 2012; Twenge, 2010). Tais diferenças podem afetar as atitudes e avaliações acerca do trabalho, influenciando as expectativas e níveis de satisfação.

Os baby boomers tendem a atribuir ao trabalho um papel de centralidade e, muitas vezes, definem-se por meio de suas carreiras (Benson \& Brown, 2011). Assim, esperava-se que a influência da ST no BES fosse maior entre os mais velhos, o que foi corroborado pelos dados e pelo fato de essa geração ter apresentado o maior valor do coeficiente de determinação (R2) da ST no BES.

\section{Considerações Finais}

Os resultados apresentados e previamente discutidos permitem um alargamento da reflexão fundamentada sobre o desafio contemporâneo da detecção, compreensão, gestão e integração da heterogeneidade/homogeneidade de perspetivas geracionais nas variáveis ST e BES nos contextos de trabalho e na sociedade. A partir dos referenciais teóricos de base, previamente apresentados, ficou saliente a plausibilidade e aplicabilidade do referencial demográfico geracional (baby-boomers; X e Y) na amostra considerada e como essa segmentação da amostra permitiu aceder às suas perspetivas valorativas específicas ao nível da satisfação laboral e explo- rar a sua relação com indicadores de AP e AN. Reforçou-se, empiricamente, o sentido da orientação teórica prevista pelos modelos de base perfilhados e referenciados - bottom-up e de spillover - no que tange ao papel efetivo de dimensões da ST na explicação de indicadores de BES adotados, mas incorporando, supletivamente, implicações particulares para uma interpretação e intervenção nos fenômenos organizacionais estudados, considerando indicadores de homogeneidade intra-geracional e heterogeneidade intergeracional.

Em particular, este estudo sinalizou diferenças entre níveis de ST e BES entre baby boomers, geração X e geração Y. Notamos que foi entre os baby boomers que ST obteve maior impacto na predição do BES, o que pode ser reflexo do papel de centralidade atribuído ao trabalho. Essa geração demonstrou também maior satisfação laboral e índices mais favoráveis dos AP e AN, o que pode indicar alguma adaptação das expectativas ao longo da vida bem como sinalizar alguma resiliência. A comparação do efeito preditor das dimensões da ST em relação ao BES sinalizou que, entre os baby boomers, a satisfação com os colegas e com o salário, além da natureza do trabalho, revelaram potencial para influenciar o AP. Tal peculiaridade pode sinalizar a importância não somente da diversificação nas tarefas, mas também da melhoria das relações interpessoais nesse grupo. Percebemos, ainda, que nessa geração o impacto da ausência de ST na predição do $\mathrm{AN}$ foi mais forte, indicando que os membros dessa geração reportaram maior frequência de $\mathrm{AN}$ quando insatisfeitos com sua vida laboral.

Entre os representantes da geração Y, o AP foi influenciado pela natureza do trabalho e pela satisfação com os colegas. Estudos sugerem que essa geração tenha perfil mais individualista (Veloso, Dutra, \& Nakata, 2008), e acreditamos que a satisfação com os colegas possa influenciar seu AP exatamente por não considerarem a necessidade de trabalhar em equipe. Esses profissionais não sentem necessidade de trabalhar em conjunto com outros e, quando isso ocorre, podem sentir maior impacto dessas relações quando não as consideram profícuas.

Já a geração X teve o AP influenciado pela natureza do trabalho e satisfação com a chefia. Esses profissionais costumam valorizar a informalidade e menor rigidez da hierarquia em seu ambiente de trabalho (Veloso et al., 2008; Veloso, Silva, \& Dutra, 2012). Desse modo, a insatisfação com a chefia teria maior potencial para influenciar seus afetos.

Os resultados indicaram um caminho para reflexão acerca dos fatores do trabalho que tendem a ser comuns entre os profissionais, mas também apontaram algumas singularidades entre os grupos geracionais, o que reforça não haver receita única que deva ser seguida quando pensamos em satisfação e bem-estar dos trabalhadores. Por vezes, um elemento que aumenta a satisfação laboral de um indivíduo pode não produzir efeitos na satisfação de outro(s) ou até mesmo aumentar sua insatisfação. Os indivíduos possuem diferentes estruturas de valores e estes podem afetar o peso que determinados elementos do trabalho, representados aqui pelas dimensões da ST, assumem em suas vidas enquanto fatores potencialmente desencadeadores de AP e AN.

Nossos resultados permitiram, também, sugerir que os efeitos das políticas organizacionais que enfatizem a ST podem, potencialmente, produzir melhorias no BES dos 
trabalhadores, o que reforça a importância do papel da gestão de pessoas em questões relacionadas com a qualidade de vida dos trabalhadores. Em um cenário cada vez mais competitivo, é fundamental que as organizações desenvolvam estratégias que permitam o incremento da produtividade e a valorização dos seus empregados, criando condições que favoreçam a satisfação profissional, o bem-estar e consequentemente estimulem o bom desempenho do trabalhador.

Esta investigação atingiu os objetivos aos quais se propôs e ofereceu contribuições significativas ao campo teórico e empírico da ST e do BES, mas, ainda assim, há limitações e prolongamentos futuros que merecem ser destacados. Apesar de termos encontrado diferenças significativas nas comparações entre as gerações consideradas, algumas características específicas como as variações no mercado de trabalho ou as diferenças no conteúdo das tarefas poderão causar influências nessas relações que, entretanto, não foram aqui nem objetivo nem objeto de estudo. Esse último ponto associa-se, também, com a conceptualização e operacionalização da noção de geração adotada. Assumindose com Hughes e O'Rand (2005) que cohorts de nascimento são frequentemente referidos como gerações, entendeu-se a geração como um grupo de pessoas que nasceu em um mesmo ano ou em um determinado intervalo etário. Essa opção de constituição das gerações, como um tipo de grupo etário, ainda que seja um critério teoricamente orientado (e.g., Eisenstadt, 2009; O'Donnell, 2001) e considerado como metodologicamente válido e exequível (e.g., Carlsson \& Karlsson, 1970), pode também ser perspetivado como introdutor de alguma limitação neste estudo. Essa opção de segmentação da amostra assentou no pressuposto de que os membros de uma geração partilham/partilharam eventos significativos e condicionalismos históricos particulares em idades similares. A experiência acumulada de uma geração particular refletirá, assim, a intersecção da vida individual e história social (Hughes \& O'Rand, 2005) ou a interação de fatores biológicos e sociais (Eisenstadt, 2009). Contudo, considera-se que, em estudos futuros, será possível incluir critérios complementares para a determinação e validação de grupos geracionais que possam ir para além da idade e que, suplementarmente a esta, permitam verificar e reforçar o caráter comum de experiências marcantes e de condições de vida (oportunidades e constrangimentos) dos considerados integrantes de uma mesma geração em um determinado espaço-tempo e, ainda, acrescentar informação de natureza qualitativa e longitudinal sobre o como estes, na sua individualidade biográfica, experienciaram e interpretaram, de forma relativamente homogênea, ou não, um mesmo tempo histórico de vida. Esse exercício metodológico complementar permitirá a adoção de um entendimento das gerações como algo que se estabelece necessariamente no e pelo tempo (cronológico) vai para além dele e poderá incorporar as vivências e experiências psicossociais e subjetivas desse mesmo tempo, que não foram valorizadas, contudo, nos objetivos da presente investigação. Outra limitação refere-se ao fato de que a avaliação do BES teve em consideração somente o componente emocional, formado pelos AP e AN.

Ainda que tenhamos enfatizado a relação entre ST e BES, notamos que a compreensão da relação entre essas variáveis não foi esgotada e que ainda há muito que se compreender quando pensamos nesses dois constructos, de forma isolada e integrada. Pesquisas futuras devem ser desenvolvidas com o propósito de se continuar a aprofundar de maneira empírica, longitudinal e qualitativa a compreensão da natureza complexa da relação entre essas duas variáveis.

\section{Referências}

Alcobia, P. (2001). Atitudes e satisfação no trabalho. In J. M. Carvalho Ferreira, J. Neves, \& A. Caetano (Eds.), Manual de psicossociologia das organizações (pp. 281-306). Lisboa: McGraw-Hill.

Andersen, S. H. (2009). Unemployment and subjective well-being: A question of class? Work and Occupations, 36(1), 3-25. http:// doi.org/10.1177/0730888408327131

Benson, J., \& Brown, M. (2011). Generations at work: Are there differences and do they matter? The International Journal of Human Resource Management, 22(9), 1843-1865. http://doi. org/10.1080/09585192.2011.573966

Bitencourt, B. M., Onuma, F. M. S., Piccinini, V. C., Moreira, L. B., \& Severo, R. B. (2014). Sentidos do trabalho para jovens de um empreendimento solidário e para trainees. Gerais: Revista Interinstitucional de Psicologia, 7(2), 142-155.

Blanchflower, D. G., \& Oswald, A. J. (2008). Is well-being u-shaped over the life cycle? Social Science \& Medicine, 66(8), 17331749 .

Bowling, N. A., Eschleman, K. J., \& Wang, Q. (2010). A metaanalytic examination of the relationship between job satisfaction and subjective well-being. Journal of Occupational and Organizational Psychology, 83(4), 915-934. http://doi. org/10.1348/096317909X478557

Brown, M. (2012). Responses to work intensification: Does generation matter? The International Journal of Human Resource Management, 23(17), 3578-3595. http://doi.org/10 $.1080 / 09585192.2011 .654348$

Bruk-Lee, V., Khoury, H. A., Nixon, A. E., Goh, A., \& Spector, P. E. (2009). Replicating and extending past personality/ job satisfaction meta-analyses. Human Performance, 22(2), 156-189. http://doi.org/10.1080/08959280902743709

Carlsson, G., \& Karlsson, K. (1970). Age, cohorts and the generation of generations. American Sociological Review, 35(4), 710-718.

Cavazotte, F. de S. C. N., Lemos, A. H. da C., \& Viana, M. D. de A. (2012). Novas gerações no mercado de trabalho: Expectativas renovadas ou antigos ideais? Cadernos EBAP.BR, 10(1), $162-180$.

Cennamo, L., \& Gardner, D. (2008). Generational differences in work values, outcomes and person-organisation values fit. Journal of Managerial Psychology, 23(8), 891-906. http:// doi.org/10.1108/02683940810904385

Cheeseman, K. A., \& Downey, R. A. (2011). Talking "bout my generation": The effect of "generation" on correctional employee perceptions of work stress and job satisfaction. The Prison Journal, 92(1), 24-44. http://doi. org/10.1177/0032885511428796

Costanza, D. P., Badger, J. M., Fraser, R. L., Severt, J. B., \& Gade, P. a. (2012). Generational differences in work-related attitudes: A meta-analysis. Journal of Business and Psychology, 27(4), 375-394. http://doi.org/10.1007/s10869-012-9259-4 
Dancey, C. P., \& Reidy, J. (2011). Statistics without maths for psychology (5th ed.). Harlow, UK: Prentice Hall.

Diener, E. (2009). Subjective well-being. In E. Diener, W. Glatzer, T. Moum, G. Sprangers, A. Mirjam, J. Vogel, \& R. Veenhoven (Eds.), The science of well-being (37th ed., pp. 11-58). Dordrecht: Springer.

Diener, E., \& Ryan, K. (2009). Subjective well-being: A general overview. South African Journal of Psychology, 39(4), 391-407.

Diener, E., Suh, E. M., Lucas, R. E., \& Smith, H. L. (1999). Subjective well-being: Three decades of progress. Psychological Bulletin, 125(2), 276-302.

Edmunds, J., \& Turner, B. S. (2005). Global generations: Social change in the twentieth century. The British Journal of Sociology, 56(4), 559-577. http://doi.org/10.1111/j.14684446.2005.00083.x

Eisenstadt, S. (2009). From generation to generation. London: Transaction Publishers.

Field, A. (2009). Discovering statistics using SPSS (3rd ed.). London: Sage. http://doi.org/10.1234/12345678

Folami, L. B., Asare, K., Kwesiga, E., \& Bline, D. (2014). The impact of job satisfaction and organizational context variables on organizational commitment. International Journal of Business and Public Administration, 11(2), 1-19.

Galinha, I. C., \& Pais-Ribeiro, J. L. (2005a). Contribuição para o estudo da versão portuguesa da positive and negative affect schedule (PANAS): I - Abordagem teórica ao conceito de afecto. Análise Psicológica, 2(23), 209-218.

Galinha, I. C., \& Pais-Ribeiro, J. L. (2005b). História e evolução do conceito de bem-estar subjectivo. Psicologia, Saúde \& Doenças, 6(2), 203-214.

García, M. G. P. de P. (2015). A web survey analysis of subjective well-being. International Journal of Manpower, 36(1), 48-67. http://doi.org/10.1108/IJM-12-2014-0237

Georgellis, Y., \& Lange, T. (2011). Traditional versus secular values and the job-life satisfaction relationship across europe. British Journal of Management. http://doi.org/10.1111/j.14678551.2011.00753.x

Georgellis, Y., Lange, T., \& Tabvuma, V. (2012). The impact of life events on job satisfaction. Journal of Vocational Behavior, 80(2), 464-473. http://doi.org/10.1016/j.jvb.2011.12.005

Goulart, P. M. (2009). O significado do trabalho: Delimitações teóricas (1955-2006). Cadernos de Psicologia Social e do Trabalho, 12(1), 47-55.

Gouveia, V. V., Fonsêca, P. N. da., Lins, S. L. B., Lima, A. V. de., \& Gouveia, R. S. V. (2008). Escala de bem-estar afetivo no trabalho (Jaws): Evidências de validade fatorial e consistência interna. Psicologia: Reflexão \& Crítica, 21(3), 464-473.

Gravetter, F. J., \& Wallnau, L. B. (2014). Essential of statistics for the behavioral sciences (8th ed.). Belmont, CA: Wadsworth Cengage Learning.

Gursoy, D., Maier, T. A., \& Chi, C. G. (2008). Generational differences: An examination of work values and generational gaps in the hospitality workforce. International Journal of Hospitality Management, 27(3), 448-458. http://doi. org/10.1016/j.ijhm.2007.11.002

Hellriegel, D., \& John W. Slocum, J. (2011). Organizational behavior. Learning (13th ed.). Mason, OH: South-Western Cengage Learning.
Henne, D., \& Locke, E. (1985). Job dissatisfaction: What are the consequences? International Journal of Psychology, 20(2), 221-240. http://doi.org/10.1080/00207598508247734

Hughes M., \& O' Rand, A. (2005). The lives and times of the baby boomers. In R. Farley \& J. Haaga (Eds.). The American people - Census 2000 (pp. 224-259). New York: Russel Sage Foundation.

Judge, T. A., \& Klinger, R. (2008). Job satisfaction: Subjective wellbeing at work. In M. Eid \& R. J. Larsen (Eds.), The science of subjective well-being (pp. 393-413). New York, NY: The Guilford Press.

Judge, T. A., \& Watanabe, S. (1994). Individual differences in the nature of the relationship between job and life satisfaction. Journal of Occupational and Organizational Psychology, 67(1 994), 101-107. http://doi.org/10.1111/j.2044-8325.1994. tb00554.x

Kehinde, O. (2011). Impact of job satisfaction on absenteeism: A correlative study. European Journal of Humanities and Social Sciences, 1(1), 25-49.

Kim-Prieto, C., Diener, E., Tamir, M., Scollon, C., \& Diener, M. (2005). Integrating the diverse definitions of happiness: A time-sequential framework of subjective well-being. Journal of Happiness Studies, 6, 261-300. http://doi.org/10.1007/ s10902-005-7226-8

Kowske, B. J., Rasch, R., \& Wiley, J. (2010). Millennials' (lack of) attitude problem: An empirical examination of generational effects on work attitudes. Journal of Business and Psychology, 25(2), 265-279. http://doi.org/10.1007/s10869-010-9171-8

Leech, N. L., Barrett, K. C., \& Morgan, G. A. (2015). IBM SPSS for Intermediate Statistics: Use and Interpretation (5th ed.). New York, NY: Routledge.

Lent, R. W., Singley, D., Sheu, H.-B., Gainor, K. A., Brenner, B. R., Treistman, D., \& Ades, L. (2005). Social cognitive predictors of domain and life satisfaction: Exploring the theoretical precursors of subjective well-being. Journal of Counseling Psychology, 52(3), 429-442. http://doi.org/10.1037/00220167.52.3.429

Locke, E. A. (1976). The nature and causes of job satisfaction. In M. D. Dunnette (Ed.), Handbook of industrial and organizational psychology (pp. 1297-1349). Chicago, IL: Rand McNally.

Lucas, R. E., \& Diener, E. (2009). Personality and subjective wellbeing. In E. Diener (Ed.), The science of well-being (37th ed., pp. 75-102). Dordrecht, Holanda: Springer.

Macky, K., Gardner, D., \& Forsyth, S. (2008). Generational differences at work: Introduction and overview. Journal of Managerial Psychology, 23(8), 857-861. http://doi. org/10.1108/02683940810904358

Magai, C. (2008). Long-lived emotions - A life course perspective on emotional development. In M. Lewis, J. M. Haviland-Jones, \& L. F. Barrett (Eds.), Handbook of emotions (3rd ed., pp. 376-392). New York, NY: The Guilford Press.

Maroco, J. (2007). Análise estatística com utilização do SPSS. Lisboa, Portugal: Edições Sílabo. Retrieved from http://books. google.pt/books?id=YrV1PgAACAAJ

Martins, M., \& Santos, G. (2006). Adaptação e validação de construto da escala de satisfação no trabalho. Psico-USF, 11(2), 195-205.

Muchinsk, P. M. (2006). Psychology applied to work (Eighth). Belmont, CA: Thomson Wadsworth. 
Murray, A. (2011). Mind the gap: Technology, millennial leadership and the cross-generational workforce. Australian Library Journal, 60(1), 54-65. http://doi.org/10.1080/00049670.201 1.10722556

Navarro, V. L., \& Padilha, V. (2007). Dilemas do trabalho no capitalismo contemporâneo. Psicologia \& Sociedade, 19(1), 14-20.

Newman, A., Nielsen, I., Smyth, R., \& Hooke, A. (2015). Examining the relationship between workplace support and life satisfaction: The mediating role of job satisfaction. Social Indicators Research, 120(3), 769-781. http://doi.org/10.1007/ s11205-014-0613-y

Nielsen, I., Smyth, R., \& Liu, Y. (2011). The moderating effects of demographic factors and hukou status on the job satisfactionSubjective well-being relationship in urban China. The International Journal of Human Resource Management, 22(6), 1333-1350. http://doi.org/10.1080/09585192.2011.559103

O’Donnell, M. (2001). Age and generation. New York: Tavistock Publication.

Parry, E., \& Urwin, P. (2011). Generational differences in work values: A review of theory and evidence. International Journal of Management Reviews, 13(1), 79-96. http://doi.org/10.1111/ j.1468-2370.2010.00285.x

Ribeiro, M. I. B. (2013). Satisfação com o trabalho: A percepção do profissional de farmácia. In Proceedings do XV Seminário Luso- Espanhol de Economia Empresarial - SLEEE 2013 (pp. 407-413). Vila Real, Portugal: Universidade de Trás-osMontes e Alto Douro.

Russell, J. E. A. (2008). Promoting subjective well-being at work. Journal of Career Assessment, 16(1), 117-131. http://doi. org/10.1177/1069072707308142

Sánchez-Sellero, M. C., Sánchez-Sellero, P., Cruz-Gonzáles, M. M., \& Sánchez-Sellero, F. J. (2014). Características organizacionales de la satisfacción laboral en españa. Revista de Administração de Empresas, 54(5), 537-547.

Satuf, C., Monteiro, S., Pereira, H., Esgalhado, G., Marina Afonso, R., \& Loureiro, M. (2016). The protective effect of job satisfaction in health, happiness, well-being and self-esteem. International Journal of Occupational Safety and Ergonomics, 1-9. http://doi.org/10.1080/10803548.2016.1216365

Schimmack, U., Diener, E., \& Oishi, S. (2009). Life-satisfaction is a momentary judgment and a stable personality characteristic: The use of chronically accessible and stable sources. In E. Diener, W. Glatzer, T. Moum, M. A. G. Sprangers, J. Vogel, \& R. Veenhoven (Eds.), Assessing well-being (39th ed., pp. 181-212). Dordrecht: Springer.
Siqueira, M. M. M. (2008). Satisfação no trabalho. In Mirlene Maria Matias Siqueira (Ed.), Medidas do comportamento organizacional: Ferramentas de diagnóstico e de gestão (pp. 257-266). Porto Alegre: Artmed.

Tabachnick, B. G., \& Fidell, L. S. (2007). Using multivariate statistics (5th ed.). Boston, MA: Pearson.

To, S. M., \& Tam, H. L. (2013). Generational differences in work values, perceived job rewards, and job satisfaction of chinese female migrant workers: Implications for social policy and social services. Social Indicators Research, 118(3), 1315-1332. http://doi.org/10.1007/s11205-013-0470-0

Traldi, M. T. F., \& Demo, G. (2012). Comprometimento, bemestar e satisfação dos professores de administração de uma universidade federal. Revista Eletrônica de Administração, $72(2), 290-316$.

Twenge, J. M. (2010). A review of the empirical evidence on generational differences in work attitudes. Journal of Business and Psychology, 25, 201-210. http://doi.org/10.1007/s10869010-9165-6

Veloso, E. F. R., Dutra, J. S., \& Nakata, L. E. (2008). Percepção sobre carreiras inteligentes: Diferenças entre as gerações $\mathrm{Y}, \mathrm{X}$ e baby boomers. In Encontro Nacional da ANPAD - EnANPAD (Vol. 23, pp. 1-16). http://doi.org/10.1016/j.rege.2015.05.001

Veloso, E. F. R., Silva, R. C. da, \& Dutra, J. S. (2012). Diferentes gerações e percepções sobre carreiras inteligentes e crescimento profissional nas organizações. Revista Brasileira de Orientação Profissional, 13(2), 197-207.

Warr, P. B. (2011). Work, happiness, and unhappiness. Mahway, NJ: Taylor \& Francis e-Library.

Young, S. J., Sturts, J. R., Ross, C. M., \& Kim, K. T. (2013). Generational differences and job satisfaction in leisure services. Managing Leisure, 18(2), 152-170. http://doi.org/10.1080/13 606719.2013 .752213

Zopiatis, A., Constanti, P., \& Theocharous, A. L. (2014). Job involvement, commitment, satisfaction and turnover: Evidence from hotel employees in Cyprus. Tourism Management, 41, 129-140. http://doi.org/10.1016/j.tourman.2013.09.013

Recebido em 07.09.2015

Primeira decisão editorial em 11.04.2017

Versão final em 08.05.2017

Aceito em 26.05.2017 